\title{
Simultaneous Distributed Acoustic and Temperature Sensing Using a Multimode Fiber
}

\author{
Yuan Mao, Senior Member, IEEE, Islam Ashry, Senior Member, IEEE, Frode Hveding, Ahmed Y. \\ Bukhamsin, Yuxi Hong, Tien Khee Ng, Senior Member, IEEE, and Boon S. Ooi
}

\begin{abstract}
Fiber optic distributed acoustic sensor (DAS) and distributed temperature sensor (DTS) are considerably important for many applications. It is challenging to design a hybrid DAS-DTS system using the same optical fiber because the operation principles of the two sensors are different. We here deploy the widespread standard multimode fiber (MMF) for simultaneous distributed acoustic and temperature sensing. In particular, we operate the MMF in a quasi-single-mode (QSM) state to simultaneously fulfill the functionality requirements of the DAS and DTS. The reported design offers simultaneous vibration and temperature monitoring with high accuracy. In particular, the DAS has a $12.98 \mathrm{~dB}$ mean signal-to-noise ratio (SNR) and the DTS offers temperature measurements with $\pm 1^{\circ} \mathrm{C}$ accuracy. This technique is significant for many industrial applications because it efficiently tackles a longstanding issue in practical implementation.
\end{abstract}

Index Terms-Acoustic sensors, Temperature sensors, optical fiber sensors, optical sensors.

\section{INTRODUCTION}

$\mathrm{O}$ PTICAL fiber sensors possess numerous advantages including immunity to electromagnetic interference, harsh environment operation, and miniature size [1], [2]. One attractive category of them is the distributed optical fiber sensors which monitor a certain parameter, such as vibration or temperature, along the entire fiber with a predetermined spatial resolution [2]. Such distributed fiber optic sensors are used in a myriad of important applications including oil and gas industry [3], real-time structural health monitoring [4], aerospace transportation [5], among others.

Optical fiber Raman-based distributed temperature sensor (DTS) and Rayleigh-based distributed acoustic sensor (DAS) have two different operation principles and system requirements [6], [7]. In particular, DTS relies on recording the Stokes and Anti-Stokes Raman backscattered signals from

Manuscript received X, 2019; revised X, 2019. This work was supported by Saudi Aramco (RGC/3/3138-01); King Abdullah University of Science and Technology (KAUST) (BAS/1/1614-01-01, GEN/1/6607-01-01, KCR/1/208101-01).

Y. Mao, I. Ashry, Y. Hong, T. K. Ng and B. S. Ooi are with the King Abdullah University of Science and Technology (KAUST), Computer, Electrical, and Mathematical Sciences and Engineering, Thuwal 23955-6900, Saudi Arabia (e-mail: yuan.mao@kaust.edu.sa; islam.ashry@kaust.edu.sa; yuxi.hong@kaust.edu.sa; tienkhee.ng@kaust.edu.sa; boon.ooi@kaust.edu.sa). F. Hveding and A. Y. Bukhamsin are with the EXPEC Advanced Research Center, Saudi Aramco, Dhahran 34464, Saudi Arabia (e-mail: frode.hveding@aramco.com; ahmad.bukhamseen@aramco.com).

Y. Mao and I. Ashry contributed equally to this work. an optical fiber. Since Raman signal is typically very weak (60-70 dB weaker than the input pump power) [8], high power is required to be launched into the optical fiber in order to have a reasonable signal-to-noise ratio (SNR). Consequently, multimode fiber (MMF) is the preferred platform for the Raman-based DTS. This is mainly because the standard MMF has a large effective area and a high threshold power of nonlinearity which can support the needed high injected pump power, without degrading the DTS system's performance. In contrast, the operation principle of the DAS is based on observing the Rayleigh backscattered signal from an optical fiber [7]. Compared with Raman signal, Rayleigh scattering is relatively stronger and does not require such high needed pump power of the DTS system [9]. Therefore, in terms of being below the fiber's nonlinearity threshold, either singlemode fiber (SMF) or MMF can be used for DAS. However, because the functionality of DAS depends on the coherent interference of the Rayleigh signals reflected by scattering centers along the fiber [7], MMF produces significant noises in DAS systems. This is because the standard MMF supports propagating a large number of modes and each mode has its own interference signature which all produce a resultant Rayleigh signal independent of vibration. As a result, MMF and SMF are ideal for DTS and DAS, respectively.

In comparison to SMFs, MMFs are widespread because MMF-based optical systems are relatively insensitive to alignment and cheaper [10]. Therefore, MMF is more commonly used than SMF, especially for short-distance applications [10]. For example, there are thousands of MMFs already installed worldwide in oil/gas wells for different purposes including distributed temperature sensing [3]. Consequently, using the already installed MMFs to simultaneously monitor temperature and vibration will be more cost-effective than installing new SMFs for vibration sensing. However, this is challenging because the typical MMF opposes the functionality requirements of the Rayleighbased DAS.

Recently, two trials have been reported in the literature for designing a hybrid DAS-DTS system [11], [12]. In particular, Muanenda et al. experimentally designed a hybrid RamanDTS and Rayleigh-DAS system based on a cyclic Simplex coding and using a SMF [11]. The coding improves the SNR of the hybrid sensing system to measure vibrations of frequencies up to $500 \mathrm{~Hz}$ and temperatures with a resolution down to $0.5{ }^{\circ} \mathrm{C}$. The other design offers hybrid distributed 
detection of vibration, temperature, and strain [12]. In this work, Rayleigh and Brillouin scattering have been simultaneously utilized, when injecting modulated pulses into a SMF. The two systems lack simplicity because of relying on laser coding. Additionally, both avoided using the widespread standard MMF.

We here tackle this challenge: designing a hybrid DASDTS using the commercially available MMF. Using a wavelength division multiplexer/demultiplexer, we separate the backscattered Rayleigh and Raman signals used for DAS and DTS, respectively. Meanwhile, light is carefully injected into the MMF to mainly excite the fundamental mode into the fiber. In other words, we set the MMF to operate in a quasisingle-mode (QSM) state. Following this approach, the used MMF satisfies the operation requirements of both DAS and DTS.

We firstly provide a quantitative performance analysis for the MMF-based DAS when the MMF works in the QSM state and when we excite a large number of modes into the MMF. Next, we compare the performances of the standard SMFbased DAS and that designed in this work using a QSMoperated MMF, when launching pulses of a high power level into the fibers. Additionally, we experimentally prove the ability of the reported hybrid DAS-DTS system to simultaneously identify locations along the MMF subjected to vibrations, measure the frequencies of these vibrations, and monitor temperature along the MMF. The work presented here would find many important applications and also can potentially help to increase the sensing range of the typical SMF-based DAS.

\section{MMF-BASED DAS}

In this section, we investigate the SNR dependency of the MMF-based DAS on the mode composition of the injected light into the MMF. The used experimental setup is shown in Fig. 1 where a narrow linewidth laser produces continuous wave (CW) light of $1535 \mathrm{~nm}$ wavelength, $16 \mathrm{dBm}$ optical power, and $100 \mathrm{~Hz}$ linewidth. The $\mathrm{CW}$ light is then modulated into optical pulses of $100 \mathrm{~ns}$ width and $20 \mathrm{kHz}$ repetition rate, using an acousto-optic modulator (AOM). Next, the modulated pulses are amplified with an erbium-doped-fiberamplifier (EDFA1) to be injected through a circulator into a 50/125 $\mu \mathrm{m}$ MMF.

In this experiment, we measure the SNR of the DAS in two cases: Launching mainly the fundamental mode or mixtures of many modes into the MMF. The fundamental mode is injected into the MMF using the conventional center-launching method in which the axes of the SMF and MMF are aligned and then the two fibers are joined via a mating-sleeve or fusion splicing [13], [14]. The center-launching method has been widely used in optical communications to improve the bandwidth-distance product in MMF systems [13], [14]. Experimentally, as shown in Fig. 1, we inject the fundamental mode into the MMF by using a SMF-based circulator and joining the circulator's second port with the MMF under test via a mating-sleeve. It is worth mentioning that similar results are obtained when joining the two fibers with fusion splicing instead of the mating-sleeve. On the other hand, a combination of many modes can be excited into the MMF through using a MMFbased circulator. This is because light is guided through freespace optics within the circulator [15] which facilitates launching many modes at the MMF-based circulator's second port and consequently at also the input port of the MMF under test.

The used MMF under test is $\sim 4 \mathrm{~km}$ long and, near its output port, we wind a $10 \mathrm{~m}$ section of the MMF around a vibrating piezoelectric transducer (PZT) cylinder driven by a radio frequency (RF) function generator. PZT cylinders are conventionally used to calibrate DAS systems because the vibration frequency of the PZT can be well controlled by the driven function generator [16]. The output intensity profile of the MMF is captured using an objective lens $(50 \times, \mathrm{NA}=0.45)$ and a near-infrared CCD camera [Fig. 1]. As the optical pulses propagate along the MMF, Rayleigh signals are backscattered

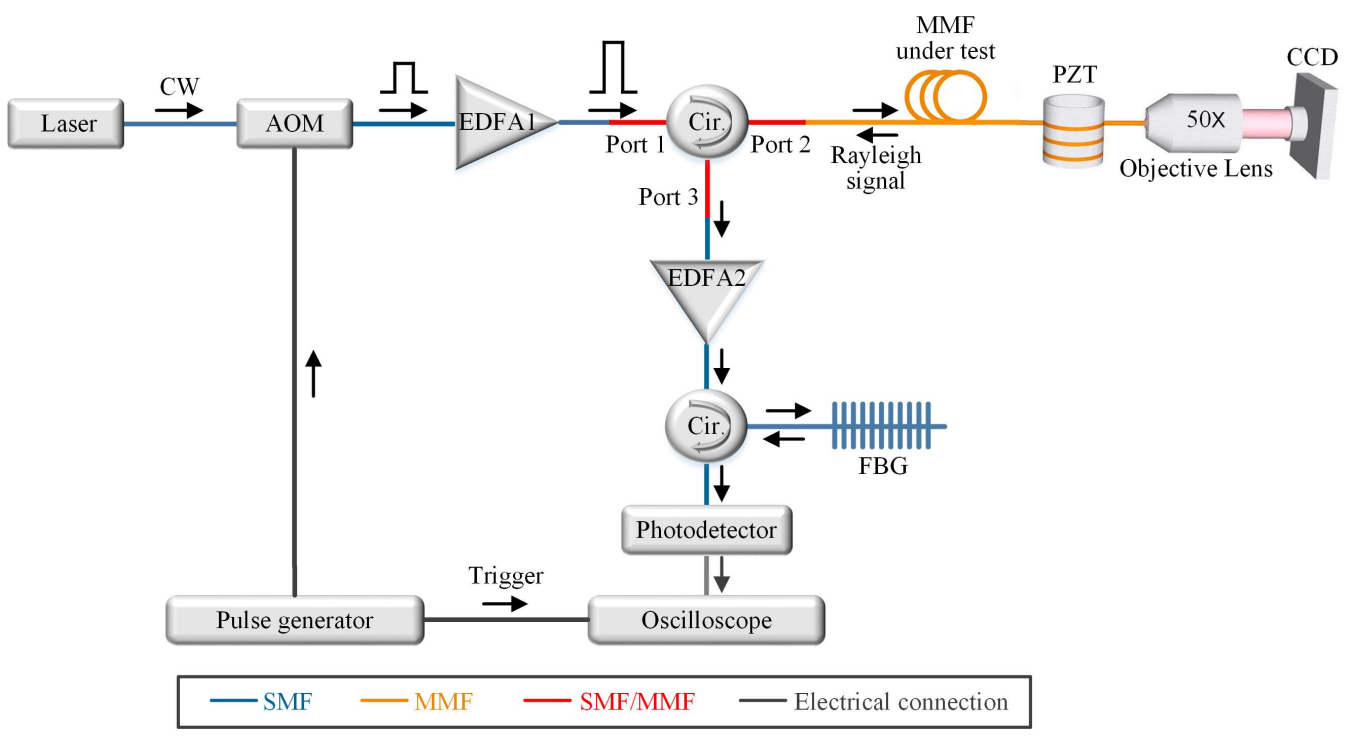

Fig. 1. Experimental setup of the MMF-based DAS. Circ., circulator. 
to be directed through the circulator towards another erbiumdoped-fiber-amplifier (EDFA2). The EDFA2's amplified spontaneous emission (ASE) noise is then discarded using a fiber Bragg grating (FBG), and finally the filtered Rayleigh signals are respectively detected and sampled using a photodetector (PD) and an oscilloscope.

When injecting an $l m$ mode into a MMF, the temporal power of backscattered Rayleigh signal received as $l ' m$ ' mode at the MMF's input port can be approximated as [17]:

$$
P_{l^{\prime} m^{\prime}}^{l m}(t)=P_{i} \gamma_{s} B_{i^{\prime} m}^{l m}, \bar{\nu} \Delta T e^{-2 \alpha \bar{v} t}
$$

where $t$ is the time, $P_{i}$ and $\Delta T$ respectively denote the power and width of an optical pulse injected into the MMF as $l m$ mode, and $\gamma_{s}$ represents the ratio of the total Rayleigh scattered power to the incident power. $\bar{v}=\left(v_{g}^{l m} v_{g}^{l^{\prime m}}\right) /\left(v_{g}^{l m}+v_{g}^{l^{\prime} m^{\prime}}\right) ; v_{g}^{l m}$ and $v_{g}^{l^{\prime} m^{\prime}}$ are respectively the group velocities of the $l m$ and $l ' m$ ' mode, and $\alpha$ is the optical attenuation coefficient. The overall capture fraction $B_{i^{\prime}{ }^{\prime}}^{l m}$, which quantifies the ratio of the scattered power into $l ' m$ ' mode to the total scattered power, is given by [17]:

$$
B_{i^{\prime} m^{\prime}}^{l m} \propto \int_{0}^{2 \pi} \int_{0}^{\infty} E_{l m}^{2}(r, \phi) E_{i^{\prime}{ }^{\prime}}^{2}(r, \phi) r d r d \phi,
$$

where $E_{l m}(r, \phi)$ and $E_{i^{\prime}{ }^{\prime}}(r, \phi)$ are the spatial near field distribution of the $l m$ and $l ' m$ ' mode, respectively. Based on Eqs. (1) and (2), when an optical pulse is injected into a MMF as $l m$ mode, the backscattered Rayleigh signal is dominated by the $l m$ mode. This is because the overall capture fraction $B_{i^{\prime}}^{l m}$ is maximized when $l m=l^{\prime} m$ '

In order to mitigate the noise in the MMF-based DAS, one should inject a single spatial mode into the MMF and simultaneously record the Rayleigh signal of the same mode. Furthermore, intermodal coupling within the MMF should also be reduced. Using the experimental setup shown in Fig. 1, we capture the intensity profiles at the MMF's output port when using the SMF- and MMF-based circulator. The intensity profiles are captured while we intentionally perturb the refractive index of the MMF to induce intermodal coupling. This is achieved via continuously blowing air flow produced by a fan onto a freely hanged $\sim 1 \mathrm{~m}$ section of the MMF. The hanged MMF section is located directly after the MMF's input port and as it shakes, because of the air flow, its refractive index is perturbed. This $\sim 1 \mathrm{~m}$ fiber section is vertically hanged and its maximum deviation from its stationary position is roughly $\pm 10 \mathrm{~cm}$, as the fan blows air towards it. Figures 2(a-d) and 2(e-h) show representative examples at different time instances to the output intensity profiles of the MMF captured when using the SMF- and MMF-based circulator, respectively. The results in Fig. 2 indicate that the conventional center-launching method mainly excite the fundamental mode in the MMF. Since the fundamental mode is non-degenerate, it is barely coupled to
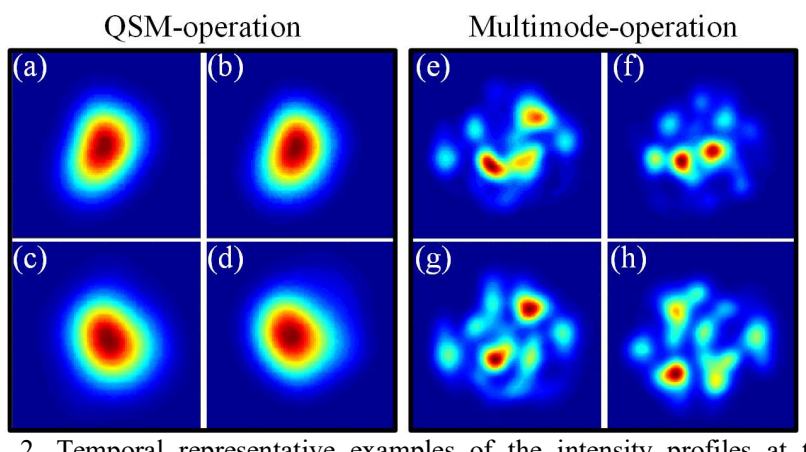

Fig. 2. Temporal representative examples of the intensity profiles at the MMF's output port when launching the fundamental mode (a-d) and many modes (e-h) into the MMF.

the other modes and consequently the fundamental mode preserves its shape until the end of the MMF [18]. When using the MMF-based circulator, in contrast, many spatial modes are launched into the MMF. Additionally, the temporal intensity profiles at the MMF's output port are unstable [Figs. 2(e-h)]. This can be attributed to the high probability of including degenerate modes excited within the MMF which can be easily coupled to each other.

Based on Eqs. (1) and (2), exciting the fundamental mode into the MMF should mitigate the noise in the DAS system, in comparison with the case of injecting many modes into the MMF. The input fundamental mode produces Rayleigh signal that is dominated by the fundamental mode. Importantly, in the backscattering direction, the SMF of the circulator's second port acts as a spatial mode filter to only pick up the fundamental mode of the Rayleigh signal and discard the other modes. Therefore, the designed system acts similarly as the SMF-based DAS, but with the presence of minor intermodal coupling which is significantly reduced when injecting the non-degenerate fundamental mode.

To experimentally prove that, we drive the PZT cylinder to vibrate sinusoidally with $5 \mathrm{kHz}$ frequency, while the fan still blows air onto the MMF to perturb its refractive index. Figures 3(a) and 3(c) show 100 consecutive Rayleigh traces recorded when injecting the fundamental mode and many modes into the $\mathrm{MMF}$, respectively. The zoom-in image shown in Fig. 3(b)/3(d) presents the temporal change of the Rayleigh intensities around the PZT location for the QSM/multimode operation case. Using the conventional differential method [7], i.e., subtracting Rayleigh traces in the time domain, we can locate the vibration location as shown in Figs. 4(a) and 4(c) when respectively exciting the fundamental mode and many modes into the MMF. Again, Fig. 4(b)/4(d) shows a zoom-in image of the differential Rayleigh signal around the PZT position for the QSM/multimode operation case. We normalize the differential signals in Fig. 4 for comparison purpose which indicates the background noise when injecting many modes into the MMF is much higher that when exciting the fundamental mode.

In order to quantify this observation for the two cases, we compare the SNR defined as the ratio between the root-meansquare (RMS) value of the differential signal at the PZT position and that along the calm fiber. Figure 5(a) shows the 

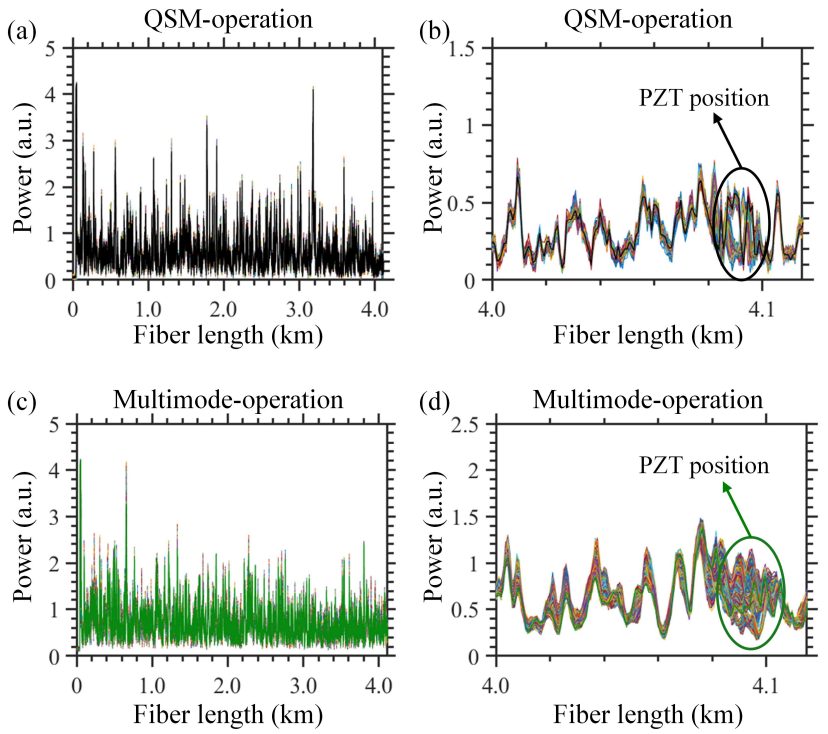

Fig. 3. 100 consecutive Rayleigh traces recorded during the QSM (a) and multimode (c) operation of the MMF. Zoom-in images of the 100 Rayleigh traces around the PZT position recorded during the QSM (b) and multimode (d) operation of the MMF.
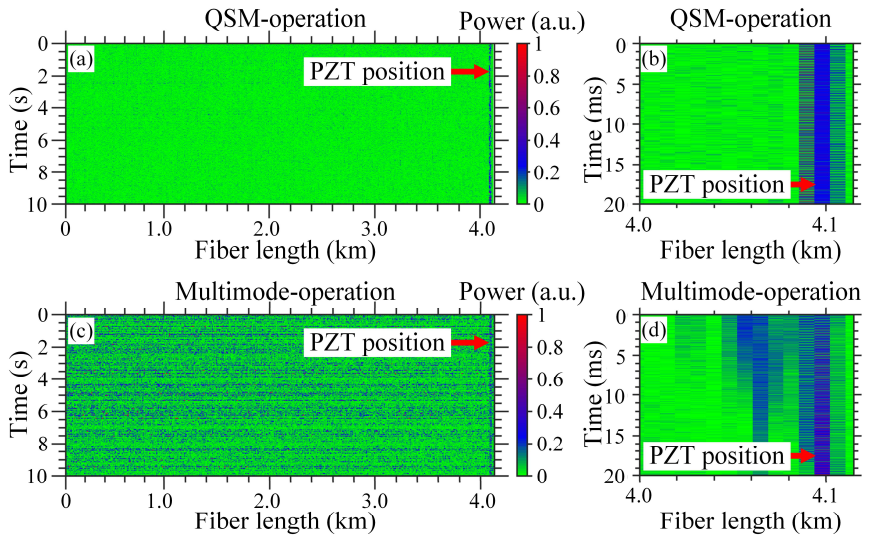

Fig. 4. Vibration position information measured during the QSM (a) and multimode (c) operation of the MMF. Zoom-in images of the vibration position information around the PZT measured during the QSM (b) and multimode (d) operation of the MMF.

temporal changes of the SNR values when injecting the fundamental mode (black circles) and many modes (green diamonds) into the MMF. Each SNR value is calculated along a $50 \mathrm{~ms}$ time period and the calculations include 300 different SNR samples [Fig. 5(a)]. This sample is sufficient to have meaningful mean and standard deviation for the SNR values. In Fig. 5(b), we present the relative frequency histograms when choosing a $1 \mathrm{~dB}$ bin for the SNR values shown in Fig. 5(a). As shown in Fig. 5(b), the Gaussian distribution fitting of the relative histogram when injecting the fundamental mode (black line) has $12.98 \mathrm{~dB}$ mean and $2.66 \mathrm{~dB}$ standard deviation. When launching many modes into the MMF, in contrast, the Gaussian distribution fitting of the relative histogram (green line) has $7.74 \mathrm{~dB}$ mean and $3.31 \mathrm{~dB}$ standard deviation. The results of Fig. 5 conclude that the reported MMF-based DAS is reliable and can be combined with the MMF-based DTS, conditioning that the MMF operates in the QSM state.
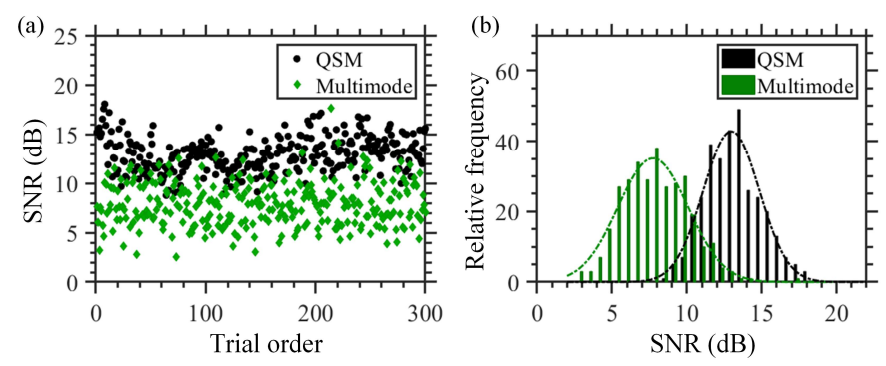

Fig. 5. (a) Temporal change of the SNR values during the QSM and multimode operation of the MMF. (b) Gaussian distribution fittings of the SNR relative frequency histograms associated with the QSM and multimode operation of the MMF.

\section{COMPARING THE PERFORMANCES OF THE SMF- AND MMF-BASED DAS/DTS}

In this section, we aim to compare the performances of the SMF- and MMF-based DAS/DTS when injecting optical pulses of high power levels. The high-power optical pulses are used to overcome the challenge of the weak Raman signals used in the DTS systems. We firstly focus on the DAS system described in the experimental setup of Fig. 1 where we here either use a standard SMF $(9 / 125 \mu \mathrm{m})$ or the 50/125 $\mu \mathrm{m}$ MMF as a fiber under test. In the two cases, light is injected into the $\mathrm{SMF} / \mathrm{MMF}$ through a SMF-based circulator. This is to avoid the loss of the core diameter mismatch when using the SMF under test, and to maximize the SNR of the DAS system when using the MMF under test, as described in Section II. As a representative example, we inject optical pulses of $P_{i}=32.24$ $\mathrm{dBm}$ individually into the two standard fibers. Figure 6(a)/6(c) shows 100 consecutive Rayleigh traces when using the $\mathrm{SMF} / \mathrm{MMF}$. For the SMF, the Rayleigh signal degrades along the fiber length. This is attributed to the SMF's nonlinearity which is dominant by the stimulated Raman scattering (SRS)
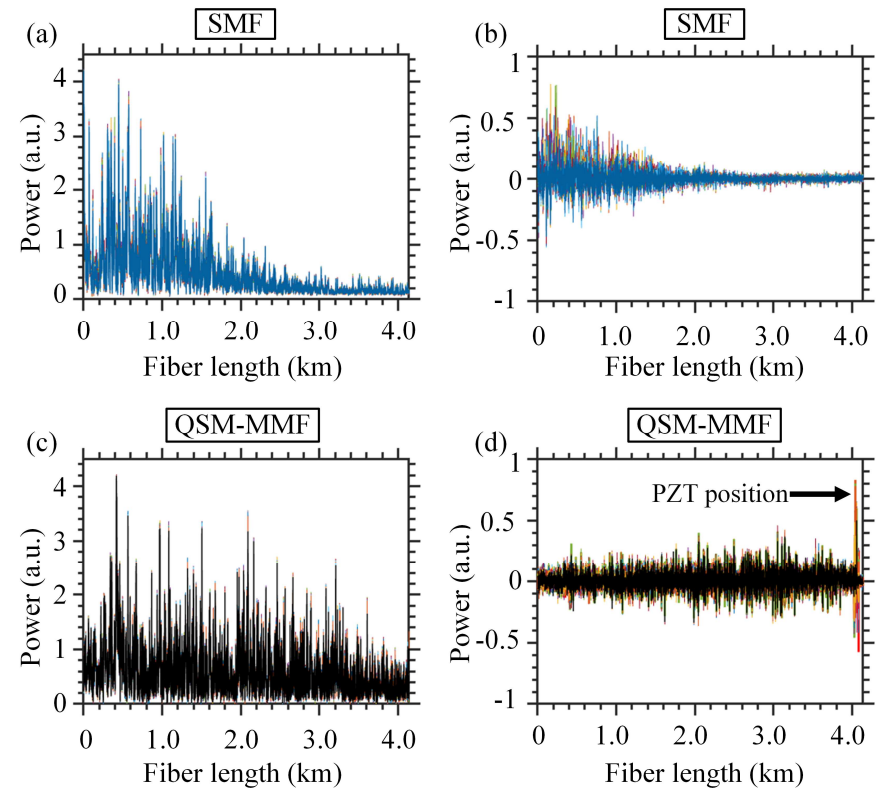

Fig. 6. (a)/(c) 100 consecutive Rayleigh traces collected when using the SMF/QSM-MMF at $32.24 \mathrm{dBm}$ input power. (b)/(d) vibration position information when using the SMF/QSM-MMF at $32.24 \mathrm{dBm}$ input power. 


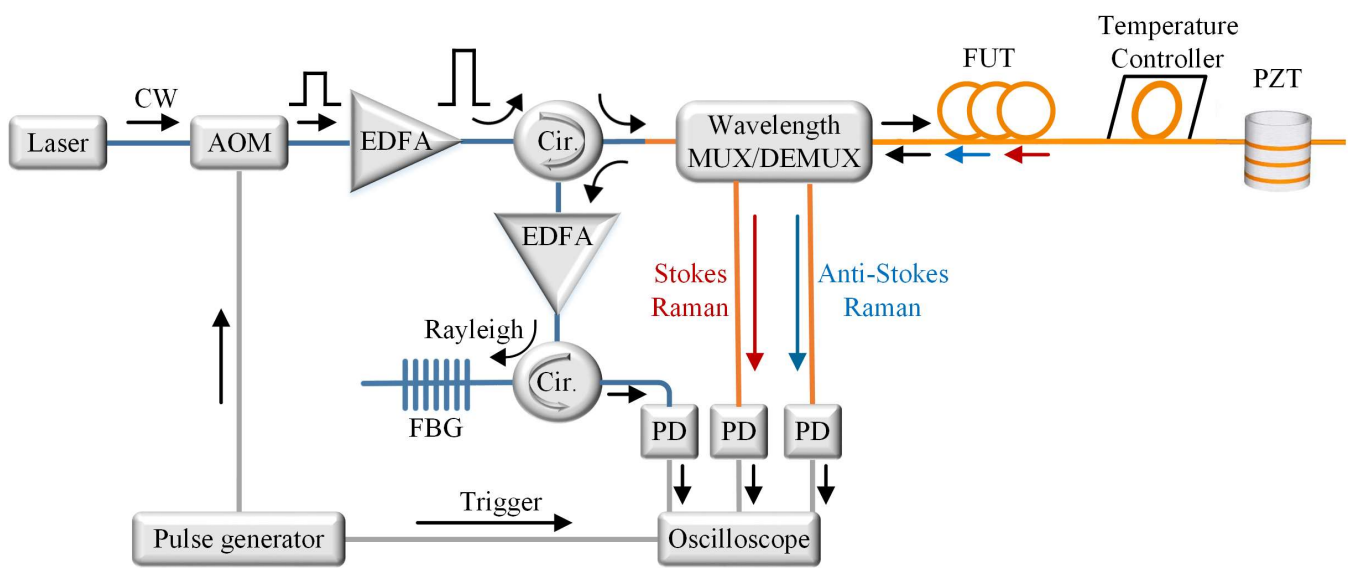

Fig. 7. Experimental setup of the hybrid DAS-DTS system. Circ., circulator; FUT, fiber under test.

for this meter-scale optical pulses [19]. In contrast, the MMF has a larger effective area and consequently a higher threshold of nonlinearity, compared with that of the SMF [19]. As a result, the Rayleigh traces keep their speckle-like profile along the MMF length [Fig. 6(c)].

Again, we drive the PZT cylinder to vibrate sinusoidally with $5 \mathrm{kHz}$ frequency when using the two fibers at $P_{i}=32.24$ $\mathrm{dBm}$. Applying the traditional differential method to locate the PZT location, Fig. 6(b)/6(d) shows the differential Rayleigh signals when using the SMF/MMF. In consistent with the results of Fig. 6(a), the SMF fails to recognize the PZT's vibration event because of the degradation of the Rayleigh signal. On the contrary, the MMF efficiently locates the vibration event even when using such high input power. These results are important since the QSM-operated MMF would be able to increase the sensing range of the typical SMF-based DAS because the MMF supports launching optical pulses of higher power.

To generalize our results, when injecting optical pulses into an optical fiber such that the peak power of the pulses is higher than the fiber's threshold of nonlinearity, the fiber cannot be reliable for vibration sensing. On the contrary, as long as the pulses' peak power is less than the fiber's nonlinearity threshold, the fiber can monitor vibrations. The sensitivity of the latter sensing case relies on many parameters, such as temporal pulse intensity fluctuation, laser phase noise and frequency drift, extinction ratio of the launched pulses, and photodetector thermal and shot noise [2], [7].

We next modify the experimental setup to further record the Stokes and Anti-Stokes Raman signals, for DTS application using either the SMF or MMF. The modified experimental setup is shown schematically in Fig. 7 where a $3 \times 1$ wavelength division multiplexer/demultiplexer (MUX/DEMUX) is added between the circulator and the fiber under test. One side of the wavelength division MUX/DEMUX includes three ports to add/drop signals of wavelengths match those of the Rayleigh, Stokes Raman, and Anti-Stokes Raman signals. The other side of the wavelength division MUX/DEMUX comprises a common port which simultaneously supports propagating the Rayleigh, Stokes Raman, and Anti-Stokes Raman signals. Since Rayleigh scattering is an elastic phenomenon, i.e., it has the same wavelength as the pump signal, the optical pulses are injected into the wavelength division MUX/DEMUX through the Rayleigh port. The optical pulses are then delivered to the fiber under test through the common port of the wavelength division MUX/DEMUX. As the optical pulses propagate along the fiber under test, Rayleigh, Stokes Raman, and AntiStokes Raman signal are backscattered, as shown in Fig. 7. The three backscattered signals can be well separated via the wavelength division MUX/DEMUX such that the Rayleigh signal is used for vibration sensing, and Stokes and AntiStokes Raman signal for temperature sensing. We use a SMFand a MMF-based wavelength division MUX/DEMUX when using the SMF and MMF under test, respectively.

We compare the Anti-Stokes Raman, and Stokes Raman signals recorded when using the SMF and MMF under test at three arbitrary high input powers such that $P_{i}=32.02 \mathrm{dBm}$, $32.24 \mathrm{dBm}$, and $32.42 \mathrm{dBm}$. Figures 8 (a) and 8 (b) respectively show the Anti-Stokes and Stokes Raman signals recorded
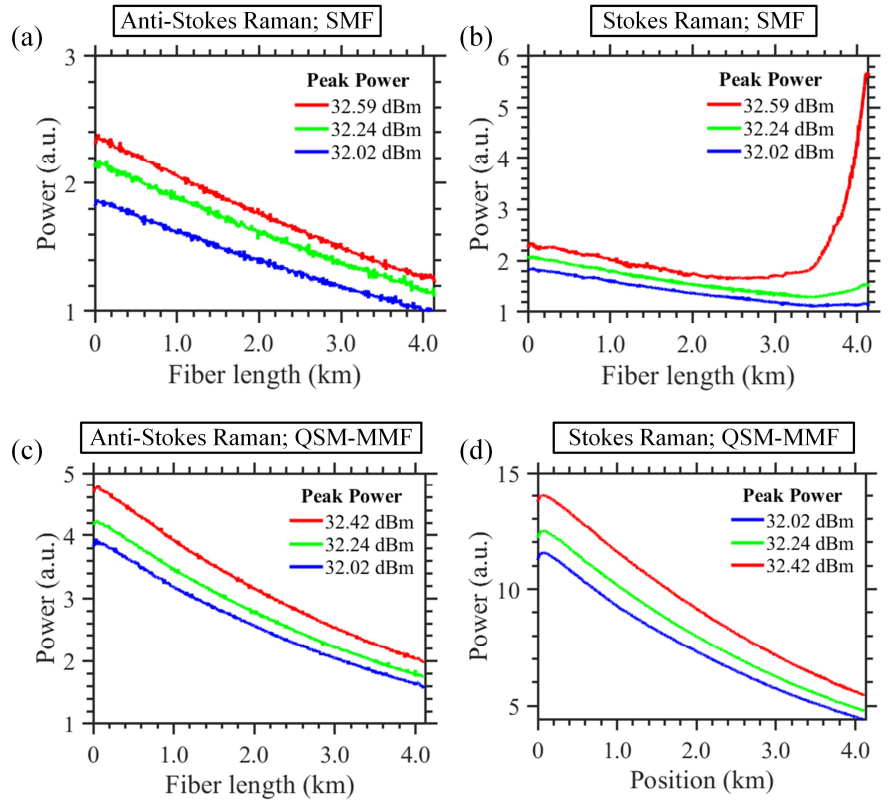

Fig. 8. (a)/(c) Anti-Stokes Raman signals when using the SMF/QSM-MMF at different input power levels. (b)/(d) Stokes Raman signals when using the SMF/QSM-MMF at different input power levels. 
when using the SMF at room temperature, for different $P_{i}$ values. Obviously, the SMF's Raman signals are distorted by the SRS, especially at higher input powers, thus unable to perform successful temperature estimation. In contrast, the Anti-Stokes and Stokes Raman signals of the QSM-operated MMF, kept at room temperature, show typical spontaneous Raman scattering feature, as shown respectively in Figs. 8(c) and 8(d). The results of this section conclude that the QSMoperated MMF is the optimum solution to simultaneously satisfy the operation requirements of the DAS and DTS systems.

It is worth mentioning that the peak powers of the injected optical pulses are controlled via tuning the pump current of the pulsed EDFA. We randomly select three different EDFA's pump currents, within various ranges, to clearly demonstrate the nonlinearity behavior of the SMF-based DTS, when injecting powers higher than the SMF's nonlinearity threshold.

\section{SimUlTANEOUS VIBRATION AND TEMPERATURE MONITORING USING THE QSM-OPERATED MMF}

In this section, we deploy the QSM-operated MMF to simultaneously provide distributed vibration and temperature sensing along the MMF length. The hybrid DAS-DTS system is shown schematically in Fig. 7 where the MMF is used as the fiber under test. Near the MMF end, we immerse a $10 \mathrm{~m}$ section of the MMF in a water bath where its temperature is controllable, and another identical fiber section is wound around the PZT cylinder [Fig. 7]. As a representative example, we again drive the PZT cylinder to vibrate with a $5 \mathrm{kHz}$ frequency. Using the Rayleigh traces recorded by our system, Figs. 9(a) and 9(b) respectively show the vibration location information and power spectrum of the PZT vibration event. These results confirm the ability of the designed hybrid DASDTS system to accurately figure out the location and frequency of the PZT vibration event.

Next, we set the water bath temperature to $\sim 7.5^{\circ} \mathrm{C}$, where this temperature is measured via a commercial thermistor, while we keep the remaining fiber at room temperature. After calibrating our system by linking the ratio between the AntiStokes and Stokes Raman signals with the temperature [6], our system measures $\sim 7.2{ }^{\circ} \mathrm{C}$ temperature at the MMF position located in the water bath [Fig. 9(c)]. The difference between the temperature measured with our system and that one measured with the commercial thermistor is acceptable because most of commercial DTS systems offer $\pm 1{ }^{\circ} \mathrm{C}$ absolute temperature error [20]. This error is mainly because of the different calibration techniques applied for the optical fiber DTS and thermistor. Similarly, we raise the temperature of the water bath to $49.8{ }^{\circ} \mathrm{C}$, as measured by the commercial thermistor, to find our DTS provides $48.3{ }^{\circ} \mathrm{C}$ [Fig. 9(d)]. Again, the difference between the two measurement systems, DTS and thermistor, is within the acceptable error range. The results shown in Fig. 9 confirm the ability of our system to provide simultaneous distributed vibration and temperature sensing along the QSM-operated MMF.
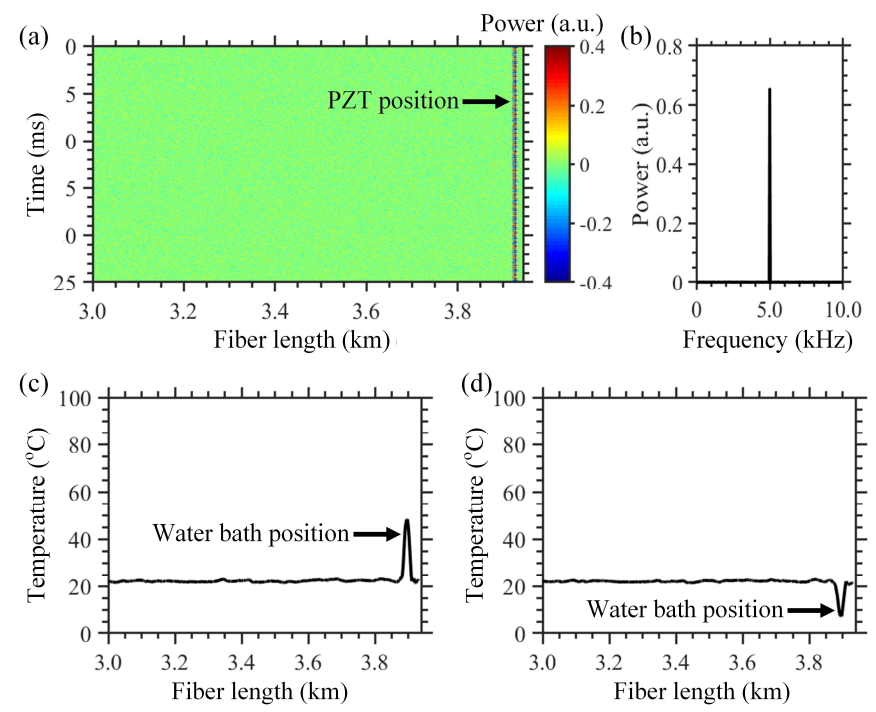

Fig. 9. Vibration position information (a) and power spectrum (b) of the $5 \mathrm{kHz}$ vibration event. Temperature distribution along the MMF when a fiber section around its end is immersed in hot (c) and cold (d) water bath.

\section{CONCLUSION}

We tackle a long-standing challenge: designing a hybrid DASDTS system using a MMF. In particular, we use the centerlaunching method to excite the fundamental mode into the MMF. We find that, even under refractive index perturbation of the MMF, the fundamental mode is barely coupled to the other modes and it maintains its spatial intensity distribution until the end of the MMF. Using this mode launching mechanism, the MMF satisfies the operation requirements of the DAS, especially because we only pick up the fundamental mode from the backscattered Rayleigh signal. The reported MMF-based DAS offers $12.98 \mathrm{~dB}$ mean SNR. We further modify the system to simultaneously extract the backscattered Rayleigh, Anti-Stokes Raman, and Stokes Raman signals from the MMF. The developed system offers simultaneous accurate vibration and temperature sensing along the QSM-operated MMF. As a representative example, the reported hybrid sensor successfully monitors vibrations of $5 \mathrm{kHz}$ frequency, and 7.2 ${ }^{\circ} \mathrm{C}$ and $48.3{ }^{\circ} \mathrm{C}$ temperatures with $\pm 1{ }^{\circ} \mathrm{C}$ accuracy.

\section{REFERENCES}

[1] B. Culshaw and A. Kersey, "Fiber-optic sensing: A historical perspective," J. Light. Technol., vol. 26, no. 9, pp. 1064-1078, 2008.

[2] X. Bao and L. Chen, "Recent Progress in Distributed Fiber Optic Sensors," Sensors (Switzerland), vol. 12, no. 7, pp. 8601-8639, 2012.

[3] T. Yamate, G. Fujisawa, and T. Ikegami, "Optical Sensors for the Exploration of Oil and Gas," J. Light. Technol., vol. 35, no. 16, pp. 3538-3545, 2017.

[4] A. Barrias, J. R. Casas, and S. Villalba, "A review of distributed optical fiber sensors for civil engineering applications," Sensors (Switzerland), vol. 16 , no. 5, pp. 748-782, 2016.

[5] S. Merlo et al., "Runways ground monitoring system by phasesensitive optical-fiber OTDR," in 4th IEEE International Workshop on Metrology for AeroSpace, MetroAeroSpace 2017 - Proceedings, 2017, pp. 523-529.

[6] X. Ma, J. Chang, Z. Wang, W. Wang, and T. Li, "Using multiple reference points in Raman based distributed temperature sensor for eliminating DC interference," IEEE Sens. J., vol. 14, no. 1, pp. 295301, 2014.

[7] X. Bao, D. P. Zhou, C. Baker, and L. Chen, "Recent Development in the Distributed Fiber Optic Acoustic and Ultrasonic Detection," $J$. 
Light. Technol., vol. 35, no. 16, pp. 3256-3267, 2017.

[8] M. A. Soto et al., "Raman-based distributed temperature sensor with 1 $\mathrm{m}$ spatial resolution over $26 \mathrm{~km}$ SMF using low-repetition-rate cyclic pulse coding," Opt. Lett., vol. 36, no. 13, pp. 2557-2559, 2011.

[9] Z. Zhang et al., "Recent progress in distributed optical fiber raman photon sensors at China Jiliang University," Photonic Sensors, vol. 2, no. 2. pp. 127-147, 2012.

[10] X. Chen et al., "Universal fiber for short-distance optical communications," J. Light. Technol., vol. 37, no. 2, pp. 389-395, 2019.

[11] Y. Muanenda, C. J. Oton, S. Faralli, T. Nannipieri, A. Signorini, and F. Di Pasquale, "Hybrid distributed acoustic and temperature sensor using a commercial off-the-shelf DFB laser and direct detection," Opt. Lett., vol. 41, no. 3, pp. 587-590, 2016.

[12] J. Zhang, T. Zhu, H. Zhou, S. Huang, M. Liu, and W. Huang, "High spatial resolution distributed fiber system for multi-parameter sensing based on modulated pulses," Opt. Express, vol. 24, no. 24, pp. 2748227493, 2016.

[13] Z. Haas and M. A. Santoro, "A Mode-Filtering Scheme for Improvement of the Bandwidth-Distance Product in Multimode Fiber Systems," J. Light. Technol., vol. 11, no. 7, pp. 1125-1131, 1993.

[14] D. H. Sim, Y. Takushima, and Y. C. Chung, "High-speed multimode fiber transmission by using mode-field matched center-launching technique," J. Light. Technol., vol. 27, no. 8, pp. 1018-1026, 2009.

[15] M. Shirasaki, H. Kuwahara, and T. Obokata, "Compact PolarizationIndependent Optical Circulator," Appl. Opt., vol. 20, no. 1, pp. 26832687,1981

[16] I. Ashry et al., "Normalized differential method for improving the signal-to-noise ratio of a distributed acoustic sensor," Appl. Opt., vol. 58, no. 18, pp. 4933-4938, 2019

[17] Z. Wang, H. Wu, X. Hu, N. Zhao, Q. Mo, and G. Li, "Rayleigh scattering in few-mode optical fibers," Sci. Rep., vol. 6, 2016.

[18] P. Lu, I. Ashry, and Y. Xu, "Adaptive Mode Control for Few-Mode Fiber Based Sensors and Sensor Networks," J. Light. Technol., vol. 35, no. 16, pp. 3562-3568, 2017.

[19] G. Agrawal, Nonlinear Fiber Optics, 5th ed. Elsevier Inc., 2013.

[20] D. Ross-Pinnock and P. G. Maropoulos, "Review of industrial temperature measurement technologies and research priorities for the thermal characterisation of the factories of the future," Proceedings of the Institution of Mechanical Engineers, Part B: Journal of Engineering Manufacture, vol. 230, no. 5. pp. 793-806, 2016.

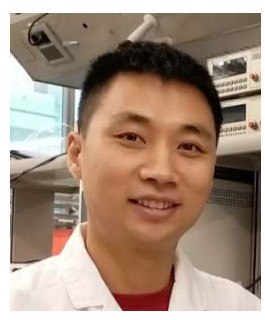

Yuan Mao received the B.S. degree in fundamental science from Tsinghua University, Beijing, China in 2007 and the $\mathrm{Ph} . \mathrm{D}$ degree in electronic and information engineering from the Hong Kong Polytechnic University, Hong Kong, China in 2013. Currently, he is a research scientist in the photonics laboratory, KAUST. His research interests include distributed fiber sensors, fiber optical communication, and wireless optical communication. He is a member of OSA, SPIE and IoP, as well as a senior member of IEEE.

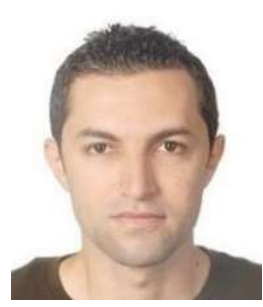

Islam Ashry received the B.S. and M.S. degrees from the University of Alexandria, Alexandria, Egypt, in 2003 and 2007, respectively, and the Ph.D. degree from Virginia Polytechnic Institute and State University (Virginia Tech), Blacksburg, VA, USA, in 2012. Currently, he is a Research Scientist at Photonics Laboratory, KAUST. His research interests include optical sensing, fluorescence dynamics, nano-patterning, plasmon sensing, nonlinear optics, fiber optics, and optical networks. $\mathrm{He}$ is a senior member of IEEE.

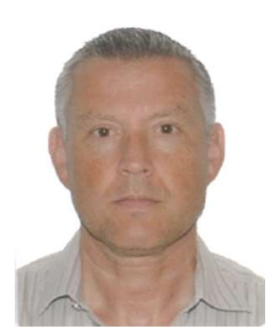

Frode Hveding holds a Master of Science degree in Petroleum Technology from the Norwegian University of Science and Technology in Trondheim, Norway.

Frode has 24 years of experience from the oil and gas industry, starting his career in Western Atlas as a Wireline Logging Engineer. After 2 years working as a Petrophysicist for Saga Petroleum in Norway, he joined Halliburton in 2000 as a Petrophysical Manager. Later he became Vice President in Ziebel, focusing on fiber optic analytics. Currently Frode works in the Production Technology Division in Saudi Aramco EXPEC Advanced Research Center, focusing on flow measurements using fiber optics.

His main areas of expertise are distributed fiber optic for downhole applications, production logging and formation evaluation, advanced data acquisition and the active use of real time information for well bore placement and geosteering.

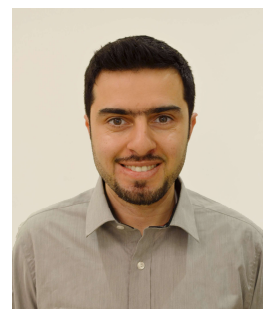

Ahmed Y. Bukhamseen is a Petroleum Engineer working with the Upstream Advanced Research Center in Saudi Aramco. He received a B.S. degree from Colorado School of Mines in 2004; and an M.S. and Ph.D degrees from Stanford University in 2009 and 2016, respectively. His current research activities cover smart well completions, production optimization, fiber optic sensing and multiphase flow metering.

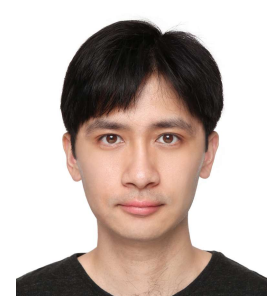

Yuxi Hong received the B.S. degree in electrical engineering from Tsinghua University, Beijing, China, in 2015 and the M.S. degree in integrated circuit engineering from Tsinghua University, Beijing, China, in 2018. He is currently pursuing the Ph.D. degree in computer science at King Abdullah University of Science and Technology, Thuwal, KSA. He was a Research Assistant with the Institute of Semiconductor at Tsinghua University, Beijing, China from 2015 to 2018, a Research Assistant with Scalable and Parallel Computing Lab, ETH Zurich from 2018 to 2019. His research interest includes machine learning, high performance computing.

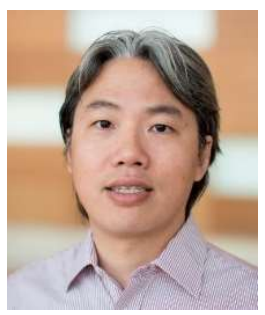

Tien Khee $\mathrm{Ng}$ received the $\mathrm{M}$. Eng. degree and the Ph.D. degree from Nanyang Technological University (NTU), Singapore, in 2001 and 2005, respectively. He was a Test Engineer with Hewlett-Packard Singapore (1997-1998), a Member of the Technical Staff with Tinggi Technologies (2004-2006), and a Research Fellow with NTU until 2009. He is a Senior Research Scientist with Ooi Group, King Abdullah University of Science and Technology (KAUST), Thuwal, Saudi Arabia. As a Co-Principal Investigator responsible for innovation in 
MBE-grown nanostructures devices and in laser devices at the KACST Technology Innovation Center on Solid-State Lighting at KAUST, he realized wide-bandgap nitride quantum confined and nanowires structures for light-emitters technology, optical wireless communication, and energy harvesting. He is a member of SPIE and IoP, as well as a Senior Member of The Optical Society (OSA), and IEEE.

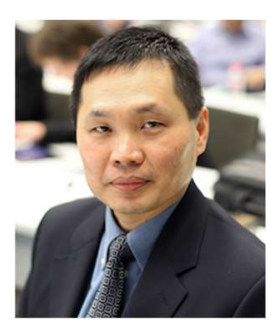

Boon S. Ooi received the Ph.D. degree from the University of Glasgow, U.K., in 1994. He is currently a Professor of electrical engineering with the King Abdullah University of Science and Technology (KAUST). He joined KAUST from Lehigh University, USA, in 2009. His recent research is concerned with the study of III-nitride-based materials and devices, and lasers for applications such as solid-state lighting, optical sensing, visible light and underwater wireless optical communications, and energy harvesting devices. He is a Fellow of the U.S. National Academy of Inventors (NAI), OSA, SPIE, and IoP, U.K. He has served on the Technical Program Committee of CLEO, IPC, ISLC, and IEDM. He currently serves on the Editorial Board of Optics Express and the IEEE photonics journal. 